31P NMR in the study of liver metabolism in vivo.

Quistorff, Bjørn; Engkagul, Arunee; Chance, Britton

Published in:

Pharmacology, Biochemistry and Behavior

Publication date:

1983

Document version

Publisher's PDF, also known as Version of record

Citation for published version (APA):
Quistorff, B., Engkagul, A., \& Chance, B. (1983). ${ }^{31} \mathrm{P}$ NMR in the study of liver metabolism in vivo.

Pharmacology, Biochemistry and Behavior, 18(1), 241-244. 


\title{
31-P NMR in the Study of Liver Metabolism In Vivo
}

\author{
BJ $Q R N$ QUIISTORFF \\ University of Copenhagen, Department of Biochemistry, Panum Institute, Copenhagen, Denmark \\ ARUNEE ENGKAGUL AND BRITTON CHANCE \\ Johnson Research Foundation, University of Pennsylvania School of Medicine, Philadelphia, PA 19104
}

\begin{abstract}
QUISTORFF, B., A. ENGKAGUL AND B. CHANCE. 31-P NMR in the study of liver metabolism in vivo. PHARMACOL BIOCHEM BEHAV 18: Suppl, 241-244, 1983.--Continuous noninvasive readout of biochemical events in body tissues is the goal of many techniques and optical and nuclear magnetic resonance (NMR) approaches seem to be at the forefront of these. Here we present a review of NMR methods for studying liver metabolism in vivo with special emphasis on methods of localizing the response appropriate to the liver itself and to the exclusion of surrounding tissues. The simplest and most direct method appears with the use of an implanted coil which enables a variety of NMR magnets to be used in this study.
\end{abstract}

Liver metabolism In vivo studies Nuclear magnetic resonance techniques

SINCE 31-P NMR is being used increasingly for study of ATP, $\mathrm{PCr}, \mathrm{Pi}$ and other phosphorylated intermediates in vivo $[1,3,6,7,9,12]$, it seems appropriate at this meeting to review briefly the potential of this technique in liver research and to give some of our recent results on liver NMR in animal models as based on our earlier studies of the perfused liver [15].

This account will focus on our methods of obtaining NMR spectra from liver in vivo and will present preliminary data on perfused liver as well as data on liver in vivo obtained with implanted transceiver coils.

McLaughlin, et al. [15] have studied the Phosphorous NMR of the perfused liver and have made some observations useful to all of those who study this organ by NMR, some of which we reproduce here in Fig. 1 (Fig. 2 of [15]). This figure illustrates the effect of repetition rate upon the spectrum of the phosphorous compounds of the perfused liver. The lower spectrum is accumulated over an interval of $1 \mathrm{hr}$ at 1 pulse every $5 \mathrm{sec}$, a usual value for phosphorous NMR studies of body organs. It shows a very high phosphate peak and ATP triplet in which the ratio $\mathrm{R}$ (see below) is 0.6 , signifying a large contribution to the A-peak of other components. If the repetition interval is decreased to $30 \mathrm{msec}$, the same data can be accumulated with a significantly enhanced signal to noise ratio in $6 \mathrm{~min}$. However, the nature of the peaks is significantly changed, the ATP peaks appear large compared to inorganic phosphate and the ratio $R$ is 02.1. Nevertheless, as McLaughlin, et al. [15] point out, the useful estimates of the time course of ATP and Pi can be made and the dynamics of changes can be followed even minute by minute, an advantage which is quite often essential to studies of metabolic control phenomena. Calibrations of the metabolites in terms of the diagram of Fig. 1 will be necessary; e.g. values obtained by analytical biochemical techniques can be used for the initial values [15]. Proportionality will in all probability exist although it should be checked periodically by analytical biochemistry when using the rapid rate of accumulation.

Salhany, et al. [17] have obtained NMR spectra of 60.7 $\mathrm{MHz}$ at a repetition rate of $1 \mathrm{sec}$ and their data, using 1500 pulses, show ATP and Pi as it is expected. The series of peaks between Pi and ATP-G would suggest greater and unexplained complexities of the liver NMR. An interesting feature of these data is that the complexity of peaks disappears when respiration is inhibited by cyanide and fails to reappear when cyanide is removed by reperfusion. Thus, while their data suggest caution in identifying the peaks in the phosphate region, the failure to reproduce these in further manipulations of the liver questions this interpretation.

Finally, Gordon et al. [10] employ "field spoiling" to eliminate contributions from non-liver tissue to the NMR signal of the surface coil placed on the abdomen and reasonably well reproduce the spectrum of a perfused liver as obtained by McLaughlin et al. [15] when they combine rapid pulsing with field spoiling.

The expectation arising from these results is that the liver will contain the usual triplet of ATP signals, no $\mathrm{PCr}$ signal and a double signal in the inorganic phosphate region which may be due to two pools of inorganic phosphate [8], or to the combination of this with signals from 2,3-diphosphoglycerate.

This communication is intended to show results obtainable from the animal in vivo with an implanted coil.

\section{METHOD}

Male Wistar rats, fasted for $24 \mathrm{hr}$, weighing $80 \mathrm{~g}$ were used. The animals were anaesthetized IP with Nembutal, 50 

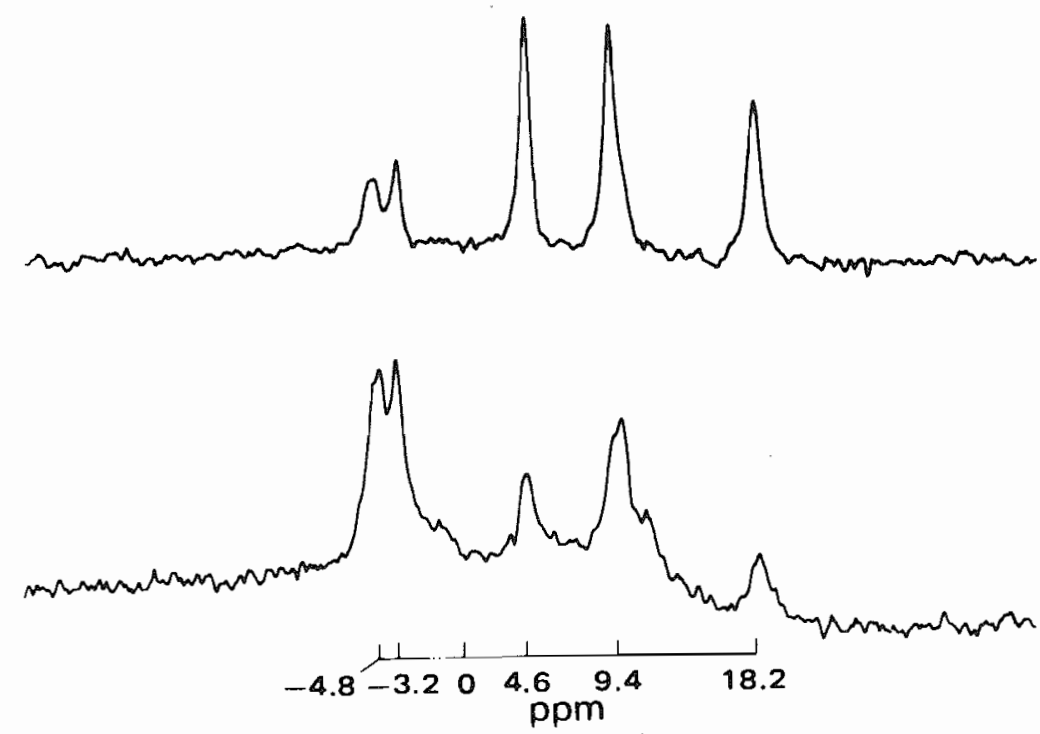

FIG. 1. 31-P NMR spectra from perfused mouse liver. (Upper) spectrum was accumulated in $6 \mathrm{~min}$ with a repetition rate of $30 / \mathrm{sec}$. (Lower) spectrum was accumulated in $1 \mathrm{hr}$ with a repetition rate of $0.2 / \mathrm{sec}$. The scale at the bottom is in ppm, measured wtih respect to an external phosphoric acid sample. The assignments are: $18.2 \mathrm{ppm}, \mathrm{B}-\mathrm{ATP} ; 9.4 \mathrm{ppm}$ A-ATP + A-ADP; $4.6 \mathrm{ppm}, \mathrm{G}$-ATP + B-ADP; $-3.2 \mathrm{ppm}$, inorganic phosphate; -4.8 $\mathrm{ppm}$, unassigned (see text). The spectra were run at $20^{\circ} \mathrm{C}$ and the line-broadening was 20 $\mathrm{Hz}$ on each spectrum. (From [15] with permission.)

$\mathrm{mg} / \mathrm{kg}$. The implanted transceiver coil was a Helmholtz type of coil with 4 turns, $15 \mathrm{~mm}$ in diameter and $28 \mathrm{~mm}$ long, made from copper wire, $1.8 \mathrm{~mm}$ in diameter, and wrapped in Parafilm to avoid direct contact with the tissue.

The coil is implanted in the abdomen via a transverse incision just below the curvature, and fitted around the liver with two turns on each side without compromising liver circulation. The incision is carefully sutured and the leads of the coil are fastened in the skin. The animal, spontaneously breathing, is further provided with an endotracheal tube in order to allow for quick changes in the inhaled gas-mixture and with a stomach tube for administration of ethanol. With the rat in place in the magnet, transitions between gas mixtures with different $\mathrm{O}_{2}$ content was possible. After each transition, a period of $10-15 \mathrm{~min}$ was allowed to establish a steady state before the scans were collected. Four humidified gas mixtures were used: Room air, $100 \% \mathrm{O}_{2} ; 10 \%$ $\mathrm{O}_{2}$; and $6 \% \mathrm{O}_{2}$.

NMR spectra were obtained at a frequency of $80.98 \mathrm{MHz}$ with Brucker a WH-360 spectrometer operated in the Fourier transform mode. Each spectrum was the sum of 10,000 free induction decays. Pulse duration was $45 \mu \mathrm{sec}$. The repetition rate was $30 / \mathrm{sec}$, as proposed by McLaughlin, et al. [15] sweep frequency $5,000 \mathrm{~Hz}$, for a total time of $333 \mathrm{sec}$. Chemical shifts are given in ppm following the UPAC convention.

\section{RESULTS}

A typical spectrum from a normal rat liver obtained with the implanted coil technique is given in Fig. 2. The following peaks may be identified as $\mathrm{G}, \mathrm{A}-$, and B-ATP at $-5.3,-10.5$ and $-19.1 \mathrm{ppm}$, respectively. The magnesium bound state of ATP in the liver is obvious from the down field shift of the

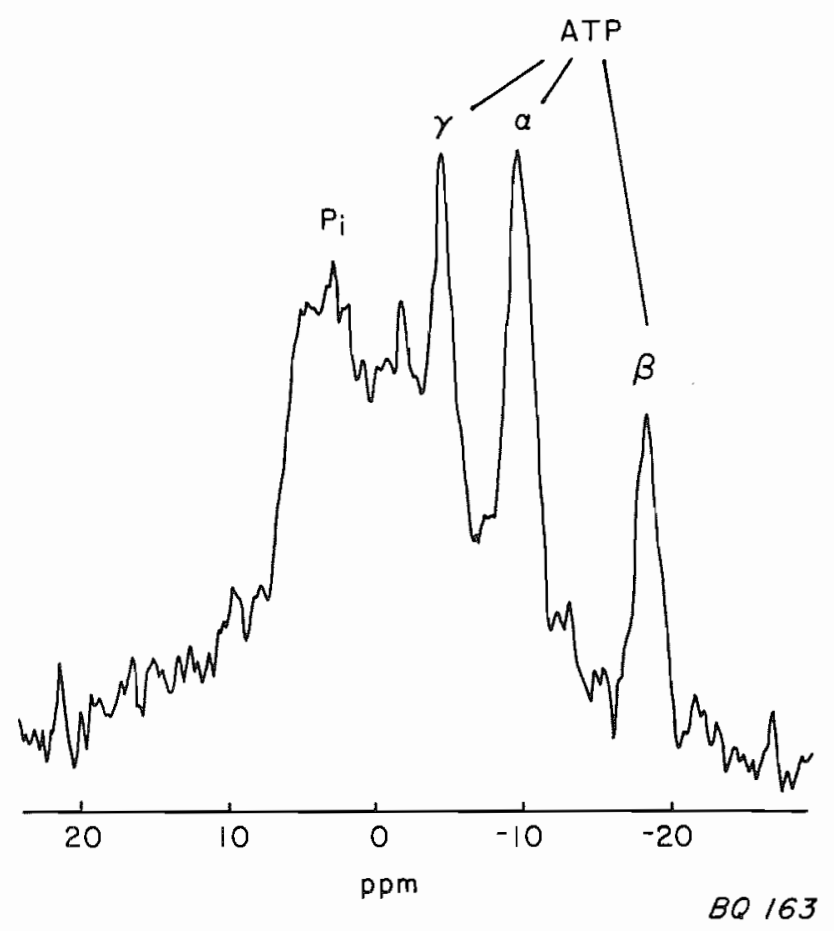

FIG. 2. In vivo 31-P NMR spectrum of rat liver. Spectrum was recorded with a Helmholtz type of coil implanted in the abdominal cavity around the liver. The animal was anesthetized and traecheostomized breathing $20 \%$ oxygen spontaneously. The ppm scale refer to an external phosphoric acid standard at $0 \mathrm{ppm}$. A linebroadening of $20 \mathrm{~Hz}$ was applied. Spectrometer parameters were as described in the text. 


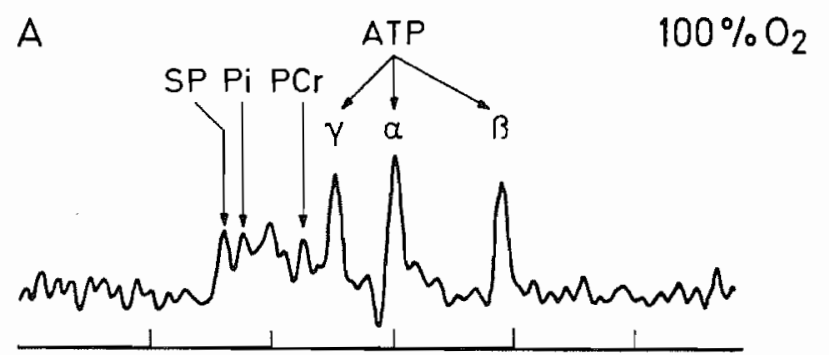

B $10 \% \mathrm{O}_{2}$

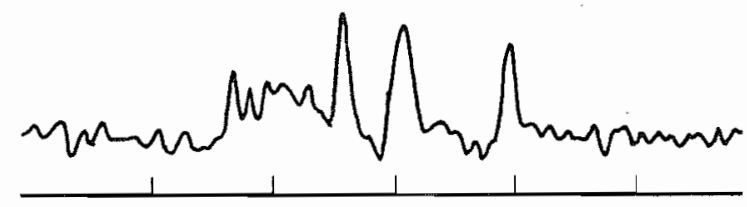

C

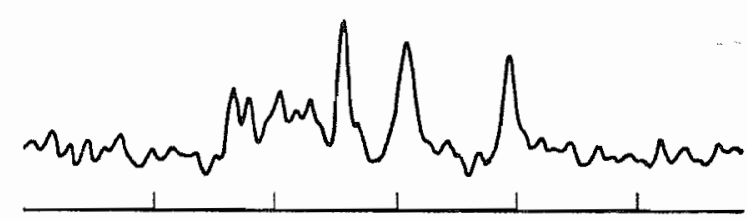

D

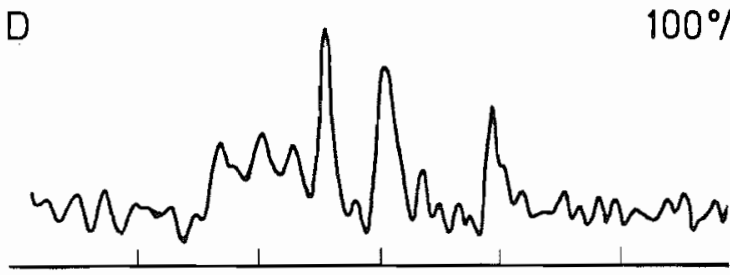

E

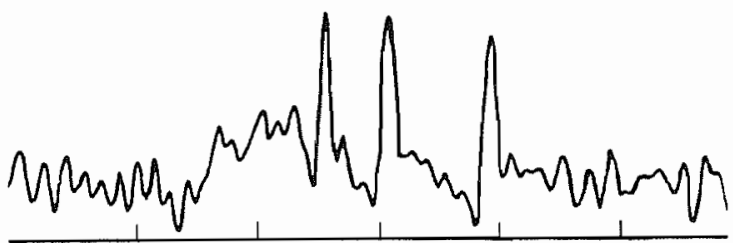

FIG. 3. In vivo 31-P NMR spectrum of rat liver. The spectra were recorded with a Helmholtz type of coil implanted in the abdominal cavity around the liver. The animal was anesthetized and traecheostomized breathing various gas mixtures spontaneously as indicated in the figure. The ppm scale refer to an external phosphoric acid standard at 0 ppm. Baseline was corrected with convolution difference, using linebroadenings of 0 and $150 \mathrm{~Hz}$.

B-peak, which in the uncomplexed state will be about -21.5 ppm [4]. Inorganic phosphate shows up as a rather broad peak at $2.1 \mathrm{ppm}$, which in some spectra actually show a splitting by $0.5-0.8 \mathrm{ppm}$ in accordance with the observation of Cohen et al. [8]. The resonance at $0.5-1.0 \mathrm{ppm}$ seems to be unaffected by hypoxia-normoxia transitions and could be glycerol-3-P-choline and glycerol-3-phosphoryl-ethanolamine [10]. The peak at 3.7-4.3 is located at the resonance for sugar phosphate and AMP. These peaks could not be resolved any further in these in vivo experiments; however, this is possi-

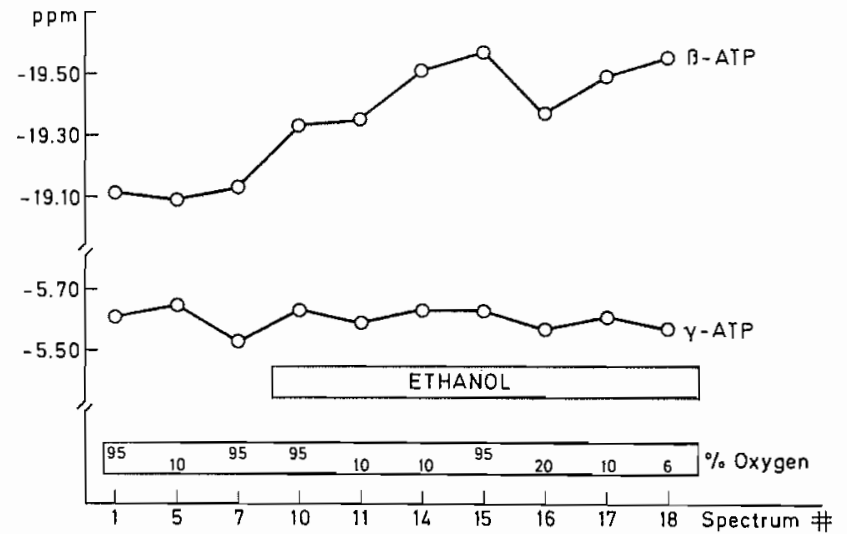

FIG. 4. Effect of ethanol on the chemical shift of B- and G-ATP resonance in vivo. The spectra were recorded as in Fig. 3. Ethanol, 1 $\mathrm{ml} 30 \%$, was administered via gastrictube.

ble following PCA extraction [16]. The very broad lines causing elevated baselines is probably phospholipids, which because of the membrane bound state does not move freely and therefore has very long relaxation times [14].

Spontaneous breathing, nembutol-anesthetized rats have been subject to a number of transitions from normoxia to hyperoxia and to hypoxia. Results typical of rapid repetition rate spectra are displayed in Fig. 3 for three values of $\mathrm{FiO}_{2}$ : $100 \%, 10 \%$ and $6 \%$. It is seen that the ATP level indicated by the $B$ peak is maintained to within $15 \%$ in these transitions, validating that the liver was adequately perfused in the interval of $\mathrm{FiO}_{2}$ from $100 \%$ to $10 \%$ to within $15 \%$. Furthermore, the complex of peaks representing sugar phosphate, inorganic phosphate and phospho-diesters shows no significant changes. The ratio of peak heights (A-ATP-B-ATP)/B-ATP is termed " $R$ " and is observed to increase in hypoxia and to decrease with the opposite transition. " $R$ " is thus more likely to be related to alterations of the $T 1$ and $T 2$ values to which the amplitudes may be very sensitive at the high repetition rate, and not to be related to ATP/ADP which has previously been studied using traditional biochemical techniques $[2,5,11,13]$, all showing decreases of ATP/ADP ratio with hypoxia. In this context it should be noted however, that Salhany et al. [17], failed to observe any ADP signal in the perfused liver treated with $\mathrm{KCN}$.

The effect of ethanol $(1 \mathrm{ml} 30 \%$ ethanol administered via gastric tube) was investigated under the conditions described above, with similar $\mathrm{FiO}_{2}$ transitions. The observations with ethanol included were as described above without ethanol. Except for the unexpected finding of a significant upfield shift of the B-ATP resonance of about $0.4 \mathrm{ppm}$, as shown in Fig. 4. It is unlikely that this is a pH effect, siince B-ATP is rather insensitive to $\mathrm{pH}$, and since there is no change in the chemical shift of $P_{1}$ upon addition of ethanol. The observed effect of alcohol could be a decrease of the intracellular magnesium ion concentration. At present however, we do not have further evidence to support this suggestion.

\section{DISCUSSION}

Two approaches have been used here to localize the NMR signal from the liver in the living animal. The first proposed by McLaughlin [15] is to increase the repetition rate of the radio frequency pulse to the point where mainly the rapid 
relaxing ATP of the liver will be observed. This has resulted in a less than perfect exclusion of other tissues near the liver. The second approach is to implant around the liver coils which are sensitive mainly to the volume between rather than the exteriorized volume. Here a combination of rapid pulsing and the implanted coil resulted in signals which contain very little if any detectable phosphocreatine and are therefore mainly referable to the liver in situ. Thus, spectra obtained with this technique may serve as a reference in future experiments on the development of surface-coil techniques with high spatial resolution.

The quality of the spectra obtained in these in vivo studies is suitable for a preliminary study, but is unsuitable for an in-depth quantitative analysis of liver metabolism and further development along these lines is required.

\section{REFERENCES}

1. Bailey, I. A., G. K. Radda and A. M. L. Seymour. The effect of insulin on myocardial metabolism and acidosis in normoxia and ischemia. Biochim Biophys Acta 720: 17-27, 1982.

2. Brosnan, J. T., H. A. Krebs and D. H. Williamson. Effects of ischaemia on metabolite concentrations in rat liver. Biochem $J$ 117: 91-96, 1970.

3. Burt, C. T., T. Glonek and M. Barany. Analysis of living tissue by phosphorous-31 magnetic resonance. Science 195: 145-149, 1977.

4. Burt, C. T., S. M. Cohen and M. Barany. Analysis of intact tissue with 31P NMR. Annu Rev Biophys Bioeng 8: 1-25, 1979.

5. Chance, B., B. Schoener, K. Krejci, W. Russmann, W. Wesemann, H. Schnitger and Th. Bucher. Kinetics of fluorescence and metabolite changes in rat liver during a cycle of ischaemia. Biochem $Z$ 341: 325-333, 1965.

6. Chance, B., S. Eleff and J. S. Leigh. Noninvasive, nondestructive approaches to cell bioenergetics. Proc Natl Acad Sci USA 77: 7430-7434, 1980.

7. Chance, B., S. Eleff, J. S. Leigh, D. Sokolow and A. Sapega. Mitochondrial regulation of phosphocreatin/inorganic phosphate ratios in exercising human muscle: A gated 31P NMR study. Proc Natl Acad Sci USA 78: 6714-6718, 1981.

8. Cohen, S. M., S. Ogawa, H. Rottenberg, P. Glynn, T. Yamane, T. R. Brown, J. R. Williamson and R. G. Schulman. 31P nuclear magnetic resonance studies of isolated rat liver cells. Nature 273: 554-556, 1978 .

9. Dawson, M. J., D. G. Gadian and D. R. Wilkie. Muscular fatigue investigated by phosphorus nuclear magnetic resonance. Nature 274: 861-866, 1978
10. Gordon, R. E., P. E. Hanley, D. Shaw, D. G. Gadian, G. K. Radda, P. Styles, P. J. Bore and L. Chan. Localization of metabolites in animals using 31-P topical magnetic resonance. Nature 287: 736-738, 1980

11. Hems, D. A. and J. T. Brosnan. Effects of ischaemia on the content of metabolites in rat liver and kidney in vivo. Biochem $J$ 120: 105-111, 1970.

12. Hoult, D. I., S. J. W. Busby, D. G. Gadian, G. K. Radda, R. E. Richards and P. J. Seeley. Observation of tissue metabolites using 31P nuclear magnetic resonance. Nature 252: 285-287, 1974.

13. Kamiike, W., S. Watanabe, T. Hasimoto, K. Taguawa, Y. Ikeda, K. Nakao and I. Kawashima. Changes in cellular levels of ATP and its catabolites in ischaemic rat liver. $J$ Biochem 91: 1349-1356, 1982.

14. McLaughlin, A. C., P. R. Cullis, J. A. Berden and R. E. Richards. 31P NMR of phospholipid membranes: Effects of chemical shift anisotropy at high magnetic field strengths. $J$ Magn Res 20: 146-165, 1975.

15. McLaughlin, A. C., H. Takeda and B. Chance. Rapid ATP assays in perfused mouse liver by $31 \mathrm{P}$ NMR. Proc Natl Acad Sci USA 76: 5445-5449, 1979.

16. Ogawa, S., H. Rottenberg, T. R. Brown, R. G. Schulman, C. L. Castillo and P. Glynn. High-resolution 31P nuclear magnetic resonance study of rat liver mitochondria. Proc Natl Acad Sci USA 75: 1796-1800, 1978.

17. Salhany, J. M., S. J. Stohs, L. A. Reinke, G. M. Pieper and J. $\mathrm{M}$. Hassing. 31-P nuclear magnetic resonance of metabolic changes associated with cyanide intoxication in the perfused rat liver. Biochem Biophys Res Commun 86: 1077-1083, 1979. 